\title{
To Be or Not to Be ... Acute Coronary Syndrome
}

\author{
Pintilie Irina*, Scridon Alina, Șerban Răzvan Constantin \\ Emergency Institute for Cardiovascular Diseases and Transplantation, Tîrgu Mureș, Romania, University of Medicine and Pharmacy, Tîrgu Mureș, Romania
}

Introduction: The association between ST segment abnormalities, elevated cardiac enzymes, and chest pain is usually a marker of acute coronary injury. However, certain other pathologies can sometimes mimic acute coronary syndromes.

Case report: A 40-year-old Caucasian male, former smoker, with no other cardiovascular risk factors, presented to the Emergency Department for typical ischemic, prolonged chest pain. The ECG demonstrated inverted T waves in leads I, II, aVL, and V3 to V6. The patient presented high cardiac necrosis markers (troponin $12.65 \mathrm{ng} / \mathrm{ml}$ ). Based on these findings, the case was interpreted as non-ST segment elevation myocardial infarction, but coronary angiography excluded the presence of significant coronary lesions. The ventriculography showed an efficient left ventricle, with mild hypokinesia of the two apical thirds of the anterior left ventricular wall. Cardiac magnetic resonance imaging demonstrated areas of hypersignal on the T2-weighted imaging sequence in the left ventricular myocardium, suggestive for acute myocarditis. The patient was started on antiplatelet, beta-blocker, and angiotensin converting enzyme inhibitor, with favorable evolution.

Conclusion: This case underlines the polymorphic appearance of acute myocarditis, which can often mimic an acute coronary event.

Keywords: myocarditis, acute myocardial infarction, cardiac magnetic resonance imaging

Received: 22 February 2016 / Accepted: 07 June 2016

\section{Introduction}

Cardiovascular diseases and particularly ischemic heart disease represent the most common cause of mortality worldwide [1]. Therefore, adequate diagnosis and treatment of these patients represent two of the most important goals in modern cardiology.

The classification of patients presented to the Emergency Department with typical chest pain relies, according to the recommendations of the European Society of Cardiology, on electrocardiographic (ECG) changes and the presence or absence of elevated levels of cardiac necrosis markers [2]. Based on these criteria, patients with chest pain and ECG changes such as persistent or transitory ST segment depression, inverted, flattened, or pseudo-normal $\mathrm{T}$ waves, or even in the absence of such ECG changes, in whom laboratory findings demonstrate elevated cardiac necrosis markers, are classified as having non-ST segment elevation myocardial infarction (NSTEMI). These patients require immediate hospital admission and specialized treatment [2].

However, pathologies other than coronary artery disease can also be associated with similar clinical scenarios, faithfully mimicking an acute coronary syndrome, including acute myocardial infarction. We report the notable case of a young patient with acute myocarditis mimicking a NSTEMI.

\section{Case report}

A 40-year-old Caucasian male, former smoker, with no personal cardiac history or ambulatory treatment, presented to the Cardiology Department of a territorial hospital, accusing two episodes of high-intensity, prolonged, constrictive chest pain the day prior to presentation, occurring

* Correspondence to: Irina Pintilie

E-mail: irina.pintilie@gmail.com at low-intensity exertion, irradiating in both upper limbs and in the interscapular region. The first episode of chest pain seized spontaneously, while the second only resolved after sublingual administration of nitroglycerine. The patient admits having abandoned smoking 5 years ago, after having smoked 15 pack-years for 15 years.

At presentation, the patient was asymptomatic, hemodynamically stable, afebrile, and had a benign physical exam. Initial ECG revealed inverted T waves in leads I, II, aVL, and $\mathrm{V}_{3}$ to $\mathrm{V}_{6}$ (Figure 1).

Laboratory analyses revealed normal complete blood count, coagulation study, and inflammatory markers levels. Cardiac necrosis markers analysis was positive for troponin I $(2.65 \mathrm{ng} / \mathrm{ml}$, normal value $<0.02 \mathrm{ng} / \mathrm{ml})$ and creatine kinase myocardial band (15.2 UI/l, normal value $<14 \mathrm{UI} / \mathrm{l})$. Liver function tests revealed elevated aspartate aminotransferase (52.9 UI/L). Lipid profile assessment revealed a total cholesterol of $129.7 \mathrm{mg} / \mathrm{dl}$, a high-density lipoprotein (HDL) cholesterol of $48.6 \mathrm{mg} / \mathrm{dl}$, a low-density lipoprotein (LDL) cholesterol of $67.3 \mathrm{mg} / \mathrm{dl}$, and plasma triglycerides of $82.8 \mathrm{mg} / \mathrm{dl}$.

Based on these findings, the case was interpreted as NSTEMI. The patient was started on dual antiplatelet therapy with aspirin $75 \mathrm{mg}$ and clopidogrel $75 \mathrm{mg}$ once daily, beta-blocker (carvedilol $3.125 \mathrm{mg}$ twice daily), angiotensin converting enzyme inhibitor (ramipril $2.5 \mathrm{mg}$ / day), and statin (atorvastatin $20 \mathrm{mg} /$ day), and was directed to the Cardiology Department of our hospital for coronary angiography.

On arrival the patient was asymptomatic, hemodynamically stable, afebrile, and the cardio-pulmonary examination was unremarkable. Arterial blood pressure was 120/80 $\mathrm{mmHg}$ and heart rate was 80 beats/minute.

The patients' social history was negative for drug use, recent acute stress, or travelling abroad and he denied having 
presented a recent history of viral illness. Compared to the previous recording, the ECG revealed biphasic $\mathrm{T}$ waves in leads $\mathrm{V}_{4}$ to $\mathrm{V}_{6}$. Transthoracic echocardiography revealed an efficient, non-dilated left ventricle, with preserved systolic function (left ventricular ejection fraction 65\%), the absence of any wall motion abnormalities or valvulopathies,

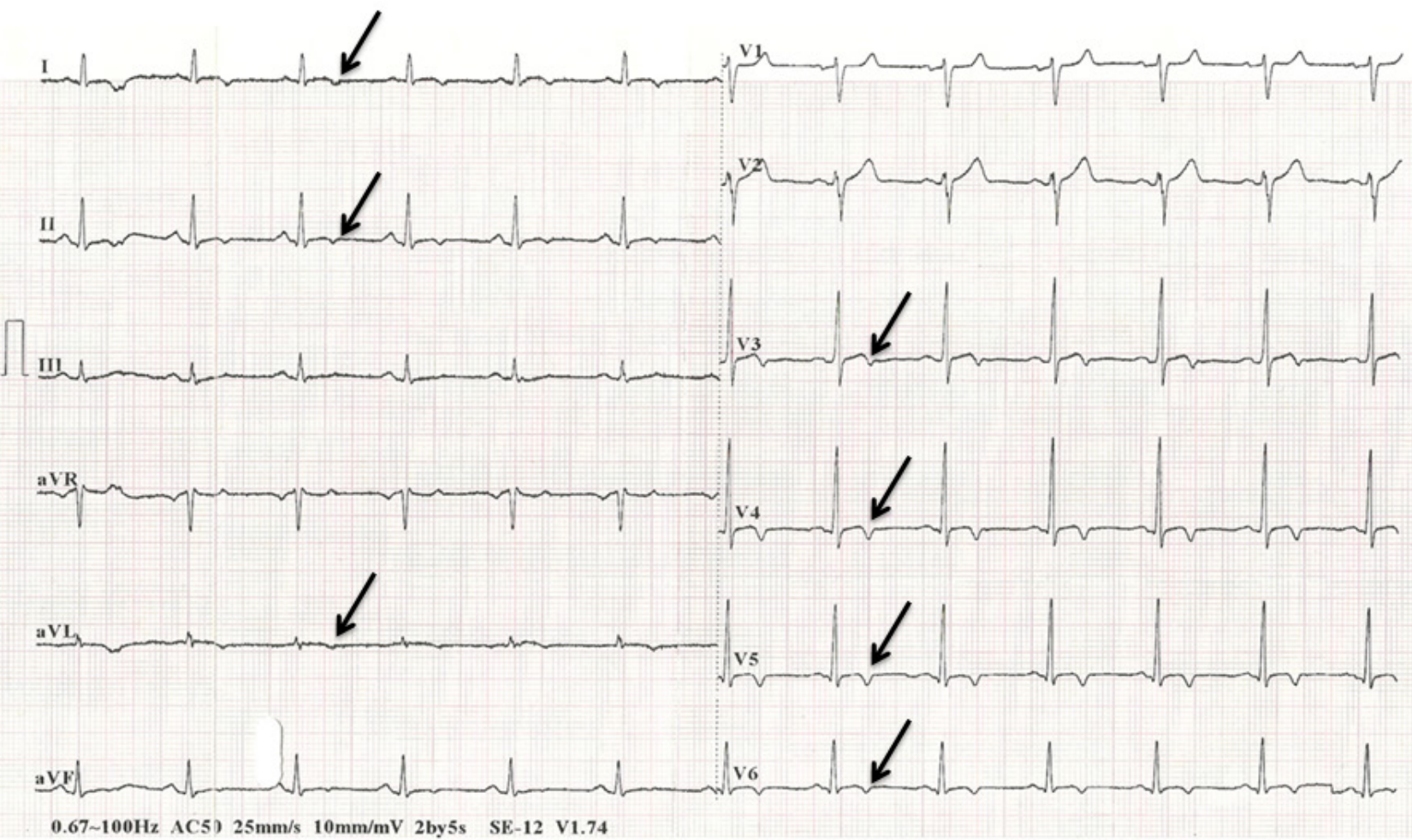

Fig. 1. Initial ECG tracing demonstrating inverted T waves in leads I, II, aVL, and V3 to V6.(arrows)

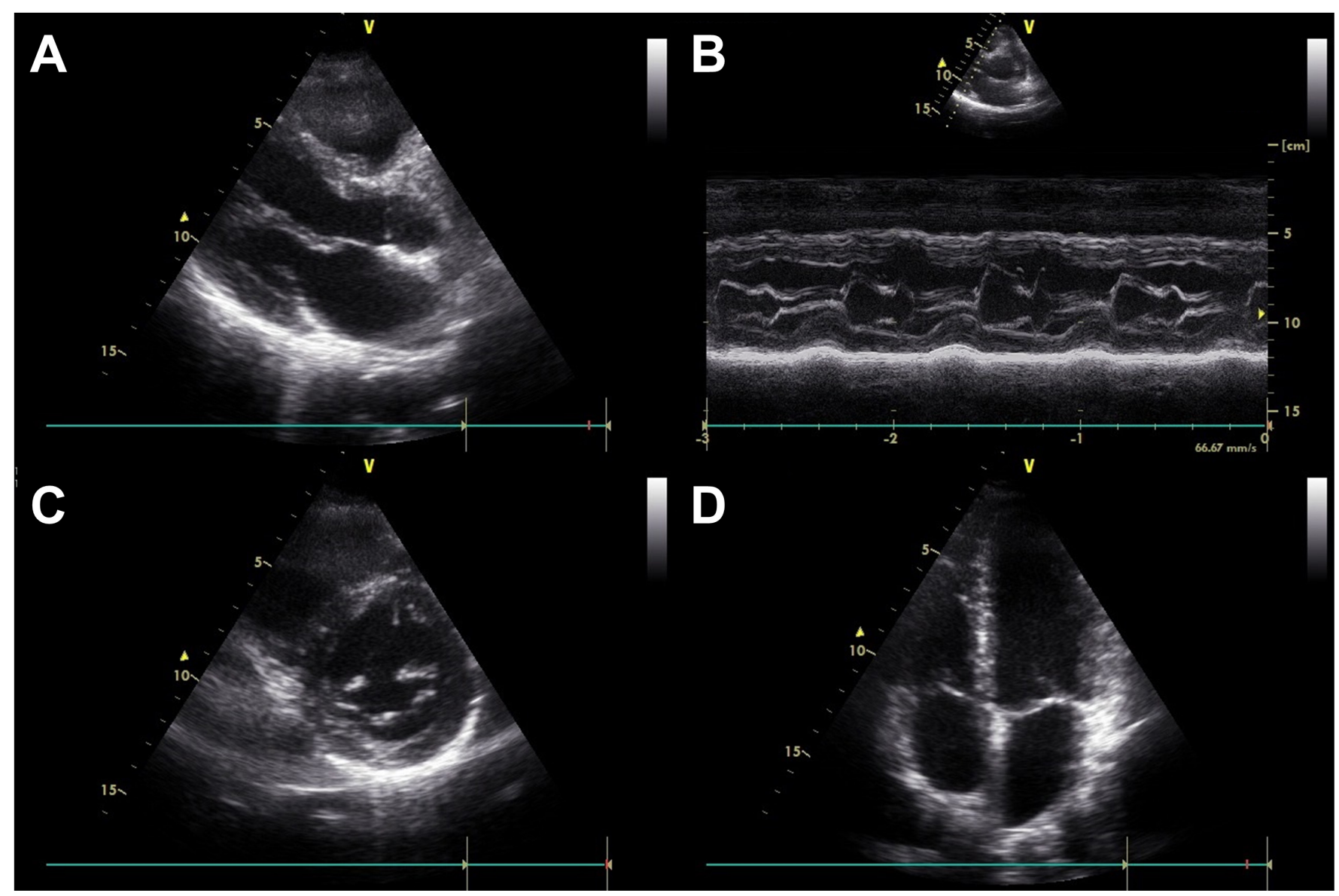

Fig. 2. Echocardiograms demonstrating a structurally normal heart: (A) B-mode, parasternal long-axis view; (B) M-mode, parasternal, longaxis view at the level of the mitral valve; (C) B-mode, parasternal short-axis view at the level of the mitral valve; (D) apical, 4-chambers view 
no pericardial thickening or effusion, and no signs of pulmonary hypertension (Figure 2).

Coronary angiography (Figure 3) revealed coronary arteries with slow flow, but no significant lesions could be observed in any of the epicardial coronary arteries. Ventriculography revealed an efficient left ventricle, with mild hypokinesia of the two apical thirds of the anterior left ventricular wall (Figure 4).

Two weeks later, cardiac magnetic resonance imaging (CMRI) was performed. Cine images revealed a left ventricle with normal volumes and systolic function (left ventricular ejection fraction 61\%), with a mass index at the upper limit of the normal range. T2-weighted imaging revealed high-intensity signal areas within the left ventricular posterior and lateral walls (Figure 5, arrows), strongly suggestive of acute myocarditis.

The patient was administered a beta-blocker (bisoprolol, $5 \mathrm{mg} /$ day) and an angiotensin converting enzyme inhibi- tor (ramipril $2.5 \mathrm{mg} /$ day) and he remained asymptomatic during hospital stay, without any signs of heart failure or arrhythmic events.

At three and six months follow-up the patient remained asymptomatic. Echocardiographic assessment confirmed the absence of kinetic abnormalities and the ECG revelead full resolution of all previous repolarization abnormalities.

\section{Discussions}

The differential diagnosis between acute myocardial infarction and acute myocarditis often represents a real clinical challenge. The present case underlines the need for an integrated interpretation of all clinical and paraclinical data in order to establish a correct diagnosis, as well as the usefulness of CMRI to confirm the diagnosis of acute myocarditis in a patient with clinical, ECG and laboratory data strongly suggestive of an acute coronary syndrome, but
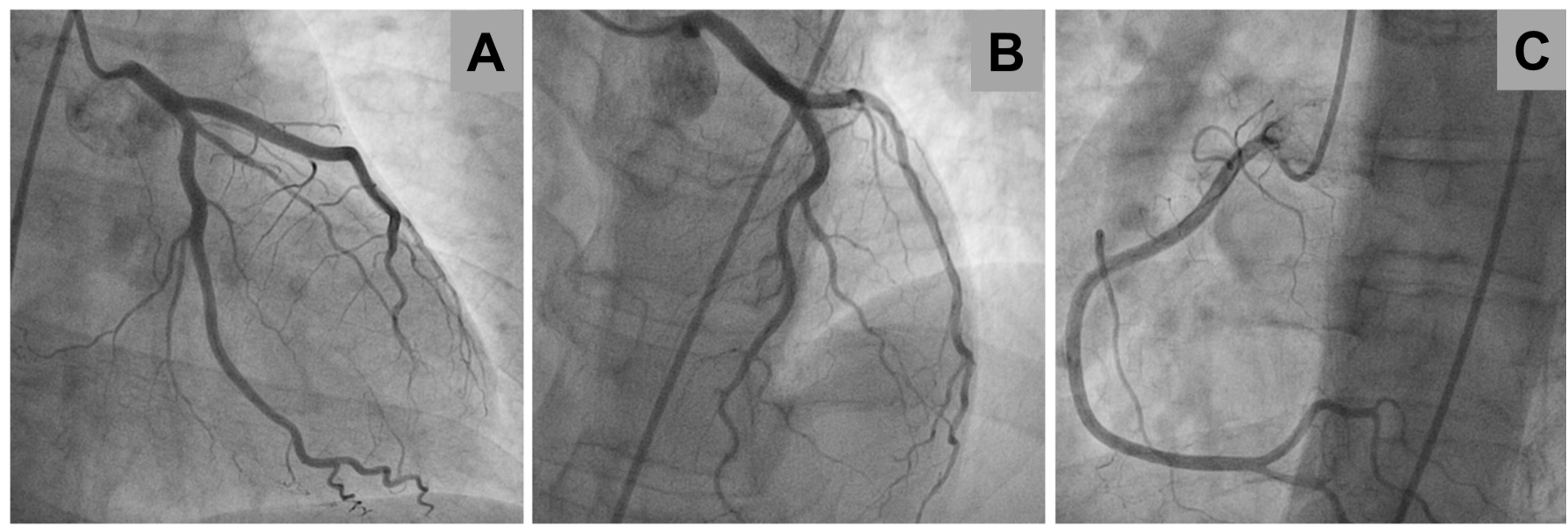

Fig. 3. Coronary angiograms of the (A) left coronary artery in right anterior oblique caudal view, (B) left coronary artery in left anterior oblique cranial view, and (C) right coronary artery in left anterior oblique view showing normal epicardial coronary arteries.
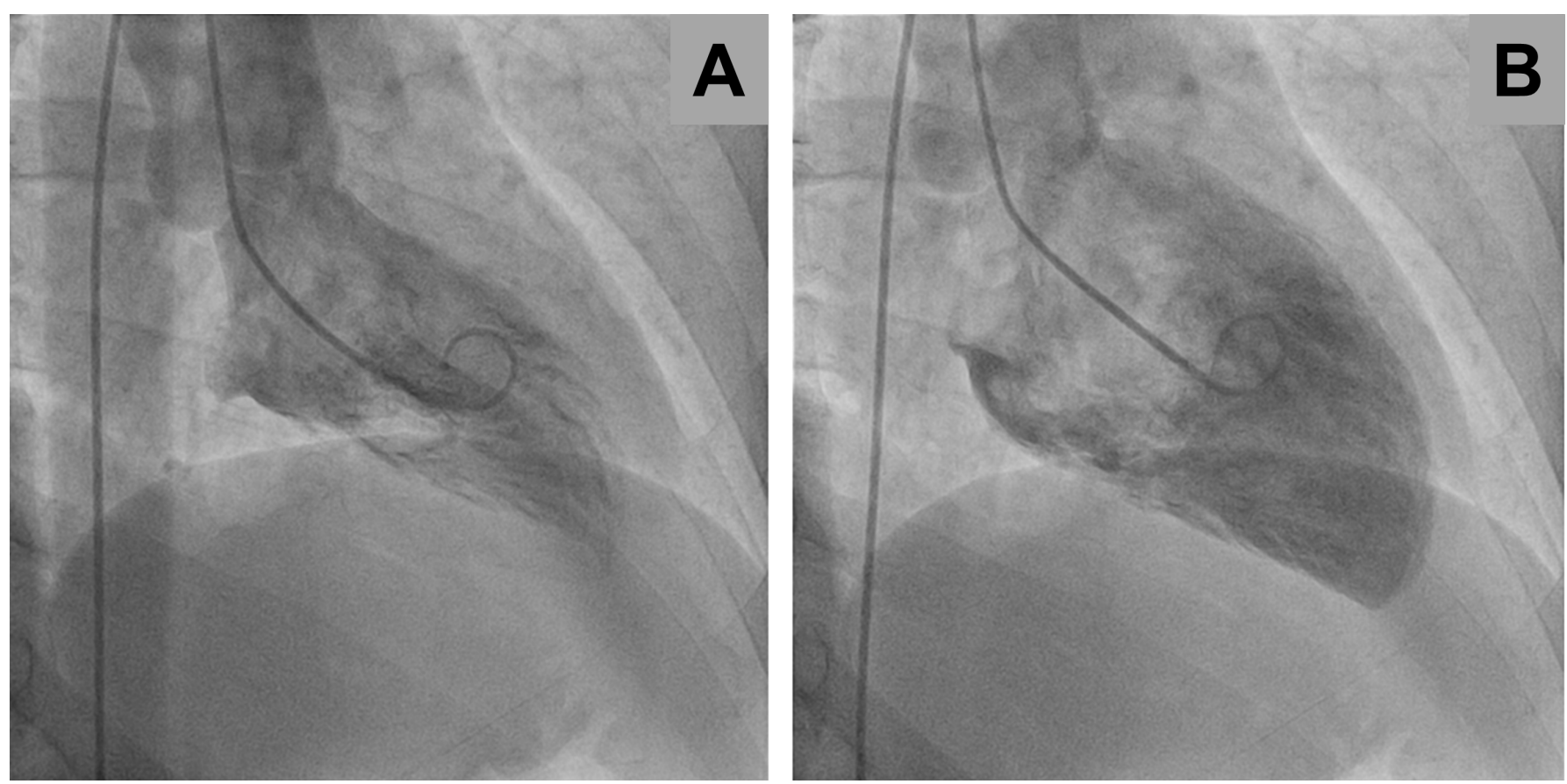

Fig. 4. Left ventriculogram in right anterior oblique view in (A) telesystole and (B) telediastole. 

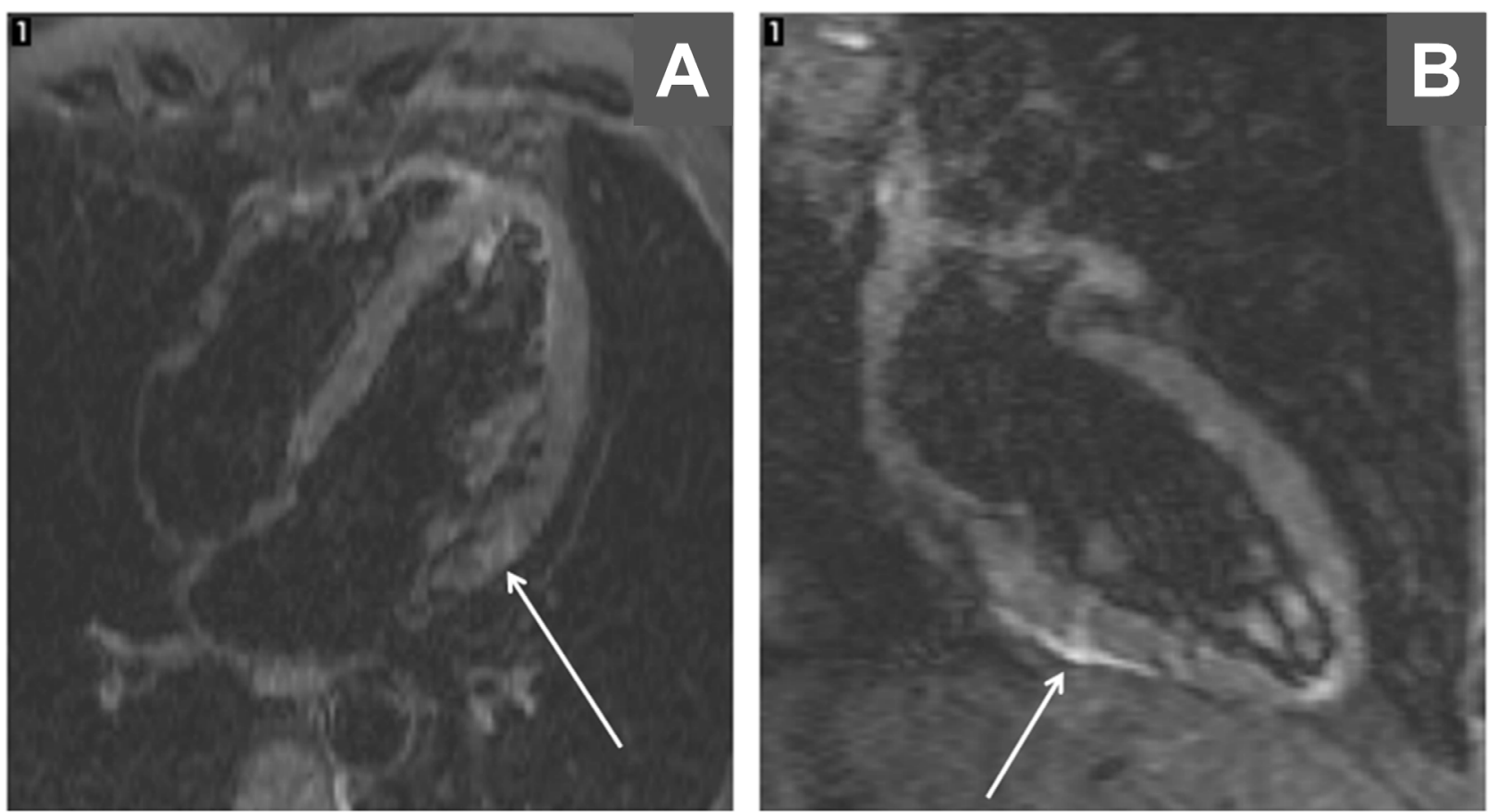

Fig. 5. Cardiac magnetic resonance images in (A) 4-chambers view and (B) two-chambers view, demonstrating diffuse patchy mid-myocardial and epicardial late gadolinium enhancement involving the left ventricular posterior and lateral walls.

in whom coronary angiography excluded the presence of coronary artery disease.

In approximately $10 \%$ of patients presented with signs and symptoms of coronary artery disease, coronary angiography reveals only minimum coronary artery lesions or even angiographically normal coronary arteries [3]. Although in the vast majority of such cases the most commonly suggested diagnosis is an acute myocardial infarction secondary to a coronary artery spasm or to a spontaneously resolved coronary artery occlusion, a clear diagnosis cannot be usually established [4].

A clinical, electrocardiographic and enzymatic picture similar to that seen in these patients can also be observed in patients with acute myocarditis. In such cases, a number of criteria can be used to identify patients with a high probability of myocarditis, including the presence of diffuse ECG changes, extended beyond the distribution territory of a coronary artery, absence of segmental ventricular wall motion abnormalities or the presence of global ventricular hypokinesia in echocardiography or ventriculography [5]. A history of recent viral infection, use of drugs or toxins, and the absence of risk factors for coronary artery disease can also lead towards a diagnosis of myocarditis.

Myocardial biopsy represents the gold standard for establishing a diagnosis of acute myocarditis. However, this investigation is not routinely used [6]. Its capacity to establish the correct diagnosis highly depends on the quality of the sample; false-negative results appear inevitably when the affected area cannot be included in the biopsy sample. Moreover, one should not forget that myocardial biopsy is an invasive procedure, involving a number of associated risks, depending on the operator's experience, the clinical status of the patient and the access site [7]. Additionally, performing a biopsy in areas suspected of ischemia is not recommended [8].

Despite the clinical presentation in favor of an acute coronary syndrome, coronary angiography excluded the presence of coronary disease. Hence, we decided to perform a CMRI. This investigation is able to assess the characteristics of the myocardial tissue in vivo and to differentiate between acute myocarditis and acute myocardial infarction in the absence of significant angiographic lesions (determined either by a temporary occlusion of a coronary artery followed by spontaneous reperfusion or by a prolonged coronary spasm). In the present case, CMRI allowed us to exclude an ischemic injury and to confirm the diagnosis of acute myocarditis.

Establishing the correct diagnosis changed the patient's treatment over the long term. In the absence of an acute coronary syndrome, the patient no longer had an indication for dual antiplatelet therapy. Moreover, since the patient did not present any significant coronary lesions or dyslipidemia, statin treatment was halted.

With the exception of hemodynamically unstable patients and those presenting with heart failure, for which a series of therapeutic recommendations are now available, there is no consensus regarding the management or the duration of treatment in patients presenting with myocarditis, who are hemodynamically stable, with preserved left ventricular ejection fraction and without any signs of heart failure. In this case, a beta-blocker (bisoprolol $5 \mathrm{mg}$ once daily) and an angiotensin conversion enzyme inhibitor (ramipril 2,5 mg once daily) were chosen, with the purpose 
of preventing myocardial remodeling that may result in a future heart failure [9].

Certain authors recommend long-term follow-up of patients suffering from acute myocarditis with an initial presentation of pseudo-myocardial infarction, but with normal coronary arteries and preserved left ventricular ejection fraction [9].

\section{Conclusion}

This case underlines the role of a complete assessment of all cardiac patients, that appearances can be deceiving and that a completely different cardiac pathology can hide behind a typical acute coronary syndrome. In such cases, imaging techniques such as CMRI may prove useful tools in establishing the correct diagnosis, representing an essential step towards an adequate long-term management.

\section{Acknowledgement}

This work was supported by the University of Medicine and Pharmacy of Tîrgu Mureș Research Grant number $17800 / 1 / 22.12 .2015$.

\section{Conflict of interest}

Nothing to disclose

\section{References}

1. Guilbert JJ. The world health report 2002 - reducing risks, promoting healthy life. Educ Health (Abingdon). 2003;16(2):230.

2. Hamm CW, Bassand JP, Agewall S, et al. ESC guidelines for the management of acute coronary syndromes in patients presenting without persistent ST-segment elevation. Eur Heart J. 2011;32(23):2999-3054.

3. Iuliano L, Micheletta F, Napoli A, et al. Myocardial infarction with normal coronary arteries: a case report and review of the literature. J Med Case Rep. 2009;3:24.doi:10.1186/1752-1947-3-24.

4. Simion A, Huidu S, Stanca I, et al. Aspecte particulare de diagnostic în miocardite acute. Revista Română de Cardiologie. 2008;23:A59.

5. Dec WG. Introduction to clinical myocarditis. In Myocarditis. From bench to bedside. Ed: Cooper LT. Totowa: New Jersey' Humana Press Inc 2003;257-281.

6. Angelini A, Calzolari V, Calabrese F, et al. Myocarditis mimicking acute myocardial infarction: role of endomyocardial biopsy in the differential diagnosis. Heart. 2000;84(3):245-250.

7. Cooper LT, Baughman KL, Feldman AM, et al. The role of endomyocardial biopsy in the management of cardiovascular disease. A Scientific Statement from the American Heart Association, the American College of Cardiology, and the European Society of Cardiology Endorsed by the Heart Failure Society of America and the Heart Failure Association of the European Society of Cardiology. Eur Heart J. 2007;28(24):3076-3093.

8. Laissy JP, Hyafil F, Feldman LJ, et al. Differentiating Acute Myocardial Infarction from Myocarditis: Diagnostic Value of Early-and DelayedPerfusion Cardiac MR Imaging. Radiology. 2005;237(1):75-82.

9. Caforio AL, Pankuweit S, Arbustini E, et al. Current state of knowledge on aetiology, diagnosis, management, and therapy of myocarditis: a position statement of the European Society of Cardiology Working Group on Myocardial and Pericardial Diseases. Eur Heart J. 2013;34(33):26362348. 